\title{
Using BIM to follow up milestones in a project plan during the design phase
}

\author{
Ø. Mejlænder-Larsen \\ Norwegian University of Science and Technology, Norway
}

\begin{abstract}
Traditionally, progress in construction projects is done by manually reporting status on activities in a project plan, very often based on subjective evaluation. This increases the possibility to report different status than actual progress, especially in large and multidisciplinary construction projects with high complexity. Could the project plan be related to a building information model (BIM) and could project progress reported directly from the BIM lead to a more accurate, consistent and coordinated status?

This paper assesses how object status in the BIM can be related to milestones in a project plan. Findings are based on experiences from the oil and gas industry. Data is gathered from case projects in Kvaerner, a Norwegian EPC (engineering, procurement and construction) contractor. The paper examines how a project plan can be connected to a BIM, focusing on the benefits and possibilities of adding status to objects in the BIM and how project progress can be reported and visualized using BIM.

Research shows that project progress can be extracted directly from the BIM by introducing control objects, where objects in the BIM are assigned statuses that measures grade of completeness. Checklists are connected to control objects and define criteria that must be fulfilled to reach a correct quality level. Control objects are connected to activities and dated in the project plan. Status on activities can then be obtained and related to milestones, to see if the project is on schedule.

By defining control objects in the BIM and adding quality levels that measure status related to milestones, the control objects can be connected to activities in the project plan. Status on each activity related to each milestone can be obtained directly from the BIM. Instead of manual reporting, progress towards milestones in the project plan can be reported directly from the BIM.

Keywords: building information modelling, project execution model, project planning, progress visualization, quality visualization.
\end{abstract}




\section{Introduction}

When design moved from $2 \mathrm{D}$ to $3 \mathrm{D}$, there was a need to get a thorough understanding of design progress, which was not very easy by looking directly into large and complex 3D models. Kvaerner, a Norwegian EPC contractor, started using a 3D design environment (a multidiscipline and object based 3D design software integrated with a number of information systems that serves as the main source of information for engineering and construction, where the main purpose is to improve the coordination and consistency between the disciplines responsible for the design in the project (Kvaerner [1])), corresponding to building information modeling (BIM) (a digital representation of physical and functional characteristics of a facility, and a shared knowledge resource for information about a facility, that forms a reliable basis for decisions during its life cycle - from concept to demolition (NBIMS [2])) in the construction industry. They began running collision controls, as part of interdisciplinary checks (IDC) in the design phase. It took a lot of time and resulted in tens of thousands of object collisions on a regular offshore production platform (topside). The process was gradually optimized, with a categorization of clashes into hard clashes (critical clashes between objects trying to occupy the same space) and soft clashes (clashes between the obstruction volumes provided around objects for access or clearance, and not the physical object). But did it say anything about the quality of the 3D design environment (hereafter called BIM)?

Kvaerner started with the objects in the BIM and looked at how they were able to harvest status on objects that had reached a measurable level of quality. Status definitions were defined for use on objects in the BIM. When the designer had completed a defined work, the objects were given relevant status. A checklist with a number of control questions were prepared for each discipline, which focused on execution on own work and interfaces towards adjacent disciplines. When the control questions were fulfilled, they were signed off in the checklist and a higher status was achieved. Several status levels were established. Eventually Kvaerner were able to extract statuses directly from the BIM, which formed the basis for the connection towards the milestones and eventually the project plan.

In research on project progress with the use of BIM, there has been very little focus on the use of object status, especially in the design phase. Sacks et al. [3] developed a BIM-enabled system to support production planning and day-to-day production control on construction sites. Common for this and similar solutions, is visualization of project and work status, by color-coding of objects. BIM is ideal for visualizing process and is used to show information that is specifically filtered for the viewer. This includes the ability to query visible objects for their relationships with work packages and their changing status through time. Similarly, Chen and Luo [4] has defined how the BIM describes quality status in construction with different color codes. The color codes are grouped in two; before or after inspection is performed. When the relevant part of the BIM is accepted (passed), it will be marked with yellow color code. If the part of the BIM fails an 
inspection, it will be marked red. A nonconformance report that states the violation of codes that fail to deliver the consistency of design intent and construction results will be issued for corrective action.

The main focus in research on BIM and progress is related to the construction phase and the $4 \mathrm{D}$ concept, where objects are linked to construction schedules, where time represents the fourth dimension. Traditionally, a 3D model and a project schedule, which are developed separately, have been combined into a 4D model. A schedule simulator is utilized to link the objects with the related scheduling activities. The resulting $4 \mathrm{D}$ model displays the construction sequence by showing consecutive objects as a progression over the time-span of the project (Wang et al. [5]). The 4D concept has been adopted in industry and several commercial applications are available for 4D construction planning (Sacks et al. [6]).

The focus of this paper is to assess how BIM can be used to follow up milestones in a project plan in the design phase. The paper is divided into two parts. The first part of the paper introduces control objects and the use of quality levels in BIM, and how status definitions can define quality of a design. The second part focuses on how control objects in the BIM can be related to milestones in the design phase, how the milestones in the design phase are related to the project plan, and how BIM can be used to follow up activities in the project plan.

The research is qualitative, conducted as case study research. Findings are based on experiences from project execution in major oil and gas projects through Kvaerner, one of Norway's largest EPC contractors. The data has primarily been gathered from two case projects at Kvaerner. These offshore projects are delivery of offshore production platforms (topsides) in the North Sea, executed as EPC contracts, and one with engineering on a subcontract. An EPC contract in the oil and gas industry corresponds to a design-build contract in the construction industry, where the engineering and construction services are contracted by a single builder or contractor. Data has primarily been collected through relevant company and project documentation and interviews with resources in key positions. The aim is to identify findings that can be adapted to the construction industry. According to Mejlænder-Larsen [7] the more similar the oil and gas industry and construction industry are related to project execution, and more specifically on variables related to BIM, the more relevant the findings from the oil and gas industry will be towards the construction industry.

\section{BIM and design quality}

\subsection{Control objects and quality levels}

A design deliverable may be divided into detailed sets of information linked to suitable control objects for each discipline. A control object consists of either one or several similar objects or objects that are grouped together with other related objects. All control objects will achieve the same quality level in the design phase (and in subsequent phases). The grade of completeness for a control object is described by status definitions. The status numbering, name and description are 
common for all control objects. Quality level is the degree of completion an activity or deliverable have at a given time, and how far each discipline has come, or how much they have done. Quality level describes what should be the quality of a given control object from creation to completion, divided in certain steps. Each quality level shall be achieved prior to or at certain milestones. The quality level descriptions refer to status for control objects (AkerSolutions [8]). Status for each control object is illustrated with a color code, so that the BIM can display the quality level directly, using color codes on each object. In design, the main quality levels are S1 "Preliminary", S2 "Release for verification", and S3 "Frozen". See Table 1 for all status definitions in the design phase.

Table 1: Status definitions used in the design phase (AkerSolutions [8]).

\begin{tabular}{|c|c|l|l|}
\hline Status & Name & Definition & Color \\
\hline S1 & Preliminary & $\begin{array}{l}\text { Control object registered with preliminary/ } \\
\text { estimated information. }\end{array}$ & \\
\hline S2 & $\begin{array}{c}\text { Released for } \\
\text { verification/IDC }\end{array}$ & $\begin{array}{l}\text { Control object released for verification/ } \\
\text { IDC. Necessary information required for } \\
\text { the verification/IDC included. }\end{array}$ & \\
\hline S3 & Frozen & $\begin{array}{l}\text { Verification/IDC comments implemented. } \\
\text { Interface towards other control objects and } \\
\text { other disciplines frozen. }\end{array}$ & \\
\hline S4 & $\begin{array}{c}\text { Detail design } \\
\text { completed }\end{array}$ & $\begin{array}{l}\text { Detail design of control object completed } \\
\text { and approved for construction. Detailing } \\
\text { shall not affect interfaces to other } \\
\text { disciplines and control objects. }\end{array}$ & \\
\hline
\end{tabular}

The connection between control objects, quality levels and status levels are illustrated in Figure 1. The illustration shows a simple concrete structure that consists of a base slab with four columns in each corner and a top slab that rests on the columns. The entire concrete structure can be seen as one system, where all objects are mutually dependent of each other. The control object is the concrete structure. The quality level of the control object is defined using status definitions (S1-S4). To fulfill a desired quality level, in this case S3, all objects must have achieved the same status level (S3). Each status level is displayed with unique color codes. Here, all objects have status S3, except column 1, which have status S2. If there are minor adjustments needed before the column can be lifted to status S3, the control object can still obtain quality level S3, but with column 1 on Hold. This means that it will be put on a punch list with outstanding issues that must be solved.

The use of status can be applied broadly. If four status levels on quality (and progress) are defined, then these can be applied in construction projects. It is important that criteria for how far the design should have come at the different status levels (S1-S4), and that they are defined relative to something that brings the work to completion. 


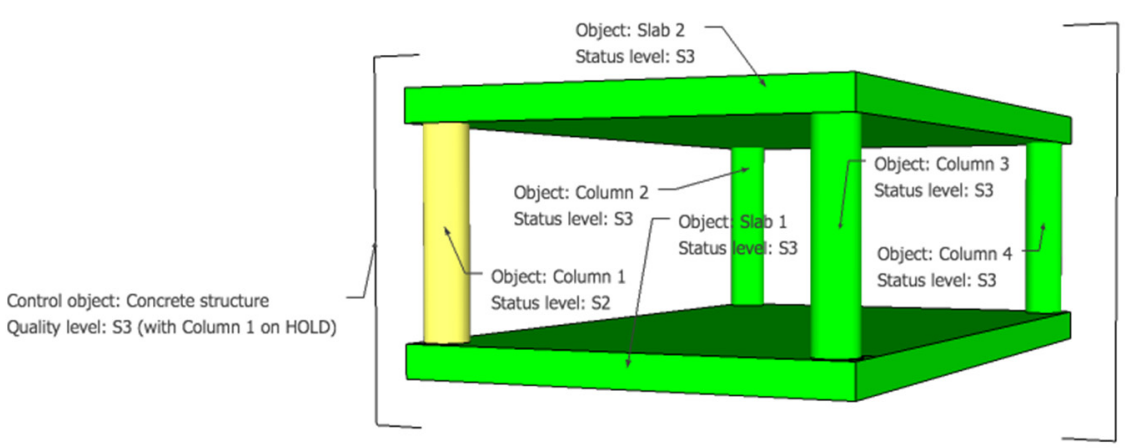

Figure 1: Illustration of a control object with quality level and status levels.

The status definitions (S1-S4) define the grade of completeness or maturity for a control object at the various milestones. Similarly, Sacks et al. [3] define 'maturity' to be a measure of the state of readiness of a work package or a task. The maturity index is displayed using color-coded symbols on task icons. Unresolved preconditions may prevent execution of the work correctly, on time and with the expected level of resource consumption.

With each control object there is a checklist that defines the requirements that must be achieved at the different quality levels. It works in the sense that when a discipline has created a design, the discipline goes through a checklist with a number of control questions for each control object. In order to demonstrate that the quality level has a certain status, (for example S3, "Frozen") all control questions shall be checked off. The discipline engineer is responsible for this. What is not done must be transferred to a punch list, so that it can be taken into account in the continuation of the work. The checklists and quality levels related to the control objects are in Kvaerner is implemented in their BIM software (AVEVA PDMS [9]). When all control questions in the checklist connected to each control object are fulfilled and checked off by a discipline, a higher quality level is achieved and status with color codes is set by the BIM software. Kvaerner has over the years developed an increasing number of control objects where the corresponding checklists are related to milestones and subsequently progress in the project plan. The reason is to try to move away from personal estimates, sometimes guesstimates, on how far each discipline has come (for example $30 \%$ complete).

Status is both quality and quantity. If a discipline have gone through the relevant checklists and fulfilled all criteria in those, and for instance achieve quality level S4 ("Detail design completed"), then relevant control objects have a quality that enables the discipline to start producing drawings for construction. At the same time, the achieved quality level is a goal, because the design will have a given number of control objects in status S4. If not all control objects are on status $\mathrm{S} 4$, the relevant discipline is behind schedule. If all control objects have status S4 the discipline is on schedule, and satisfy both quality and quantity.

Research shows that quality in the BIM can be seen through status on the objects in the model. According to Sacks et al. [6] visualization of process status 
is needed and should be displayed in a manner that can be readily understood by all, regardless of their technical knowledge. The focus has primarily been on developing a system for the construction phase, and not the design phase. The authors have defined work status icons that are shown for each location in a 3D model view for construction. This representation provides the status, the duration for which the current status has been valid, and the expected remaining work duration, where relevant.

A topside facility on a production platform often consists of up to 100,000 objects. Kvaerner cannot define control objects to follow up all of these, but must focus on the most central ones, being essential for keeping control of the project. First, Kvaerner focus on objects that are technically feasible to achieve status from. Because of the size and complexity of a topside, there will be objects that are not modeled in detail. An example is a valve, where the bolts used for fastening are not modeled, but are defined in the specification attached to the valve. Second, Kvaerner focus on objects that have several other objects connected to them, which means that the status on one object implies the same status on connected objects. An example is if a complete (frozen) pipe is located, it will assume that the steel supporting the pipe is complete. With this as a basis, Kvaerner active measures status on a minimum of $30 \%$ and up to $50 \%$ of all objects in the design phase from defined control objects in the BIM. The rest is measured manually on each activity in the project plan, where the discipline leaders set the status for each of the relevant disciplines.

\section{BIM and design progress}

\subsection{Project execution model and milestones}

The initial design phase in Kvaerner is called "System Definition" and consists of three stages (AkerSolutions [10]). The content and scope of these three stages are similar to the three stages in the design phase for the construction industry, as defined by RIBA [11]. This increases the relevance towards the construction industry. See Figure 2 for a comparison of the project stages and milestones in the two industries. In the first stage, both have focus on basis requirements for the design. The objective of stage 2A "System Design", with milestone M2A, is to identify and confirm all design basis requirements. In stage 2 "Concept design", the initial concept design is produced in line with the requirements. The second stage focuses on further development of the concept design. The objective of stage 2B "System Design and Layout Development", with milestone M2B, is to further develop the design and make sure that the overall layout is frozen. In stage 3, "Developed design", the concept design is further developed and the design work of the core designers is progressed. In the third stage, the design is finalized and frozen. The objective of stage $2 \mathrm{C}$ "Global Design", with milestone M2C, is to further develop the system and area design to a stage where all interfaces and the system design shall be frozen. In stage 4, "Technical design", the architectural, building services and structural engineering designs are further refined to provide technical definition of the project and the design work is developed and concluded. 


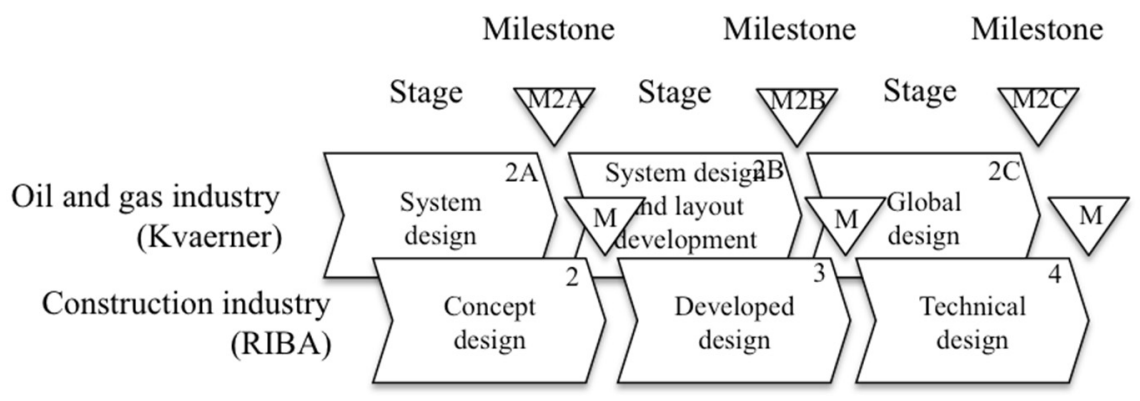

Figure 2: Comparison of project stages and milestones in the design phase.

Kvaerner has developed a project execution model (PEM), which defines what should be done, when it should be done, to what quality and at what status. The objective of a PEM is to secure predictability in project execution using a standard methodology well known to the project team. It reflects a logic sequence in critical project activities where progress and quality requirements are aligned at significant milestones to ensure predictable project execution (Kvaerner [12]).

All disciplines should know at any given time how far they are expected to have come. Knowledge of where the disciplines are is equally valuable whether they are on, ahead of or behind the milestone. If the disciplines do not measure in proportion to the status line, they in fact do not know if they have a problem and how to deal with the problem ahead. It is only when the milestone is set and is measured against it is possible to know. Experiences from the case projects show that to a milestone in the design phase, disciplines can be ahead and disciplines can be behind with their design. If a discipline has come too short it is a problem, and if a discipline has come too far, it may also be a problem. The main challenge is to take care of those behind and decide what to do with them in the continuation of the project. When the disciplines that have been behind catches up, they can influence those already finished. Much of what is done must then be redone, because the disciplines already finished have based their design on unfinished design basis, and can have made assumptions that are not correct.

The PEM controls what is the optimum picture at any given time in project progress so that all disciplines are in balance with each other. The more balance on the status line, the more likelihood for fewer design changes. The entire structure of the PEM is based on the simple reasoning that it should not be random how far each discipline has come on the various inputs on a given milestone. This is described through milestone requirements. Audits are conducted on milestones, where punch lists on what may not have been finished to the relevant milestone are developed. It will then be taken into account in the planning process in the continuation of the design, to be able to add it into future plans and to the resource picture.

The client will have contractual milestones. The milestones defined in PEM should be distributed the best way possible in the project, so that it becomes consistency between when the client claims something should be done and when 
the PEM says that it should be done. Adding milestones defined in PEM as parallel as possible with the contract milestones to the client, will avoid communicating a different message to the project team in every project. Kvaerner will always find that the client put various milestones into the contract that the client wants to measure Kvaerner on, and can add penalty or bonus to the milestones. There is also a project deadline with daily fines. Any discrepancies to each milestone must be dealt with. A punch list must be developed, so that the project plan can be considered and adjusted in relation to that. The knowledge of what have not been done is as important as the knowledge of what have been done.

\subsection{Milestones and project plan}

A project plan is created with a number of activities that should be measured on the status line related to a milestone. The activities describe control objects with relevant quality levels. Measuring begins with what is planned (forecast) status compared to actual status, to see how it complies. This is handled in regularly (weekly) meetings in Kvaerner. If the control objects should have been on quality level S4 (for example) on a given date (for milestone M2C all should be on status S4), the relevant disciplines (piping, electrical, etc.) can be chosen to see where they are behind, where they are on, and where they are ahead.

There are approximately 30,000 activities in the project plan needed to build a topside. Gradually, through experience, Kvaerner have found out what the content should be, how far the disciplines should have come and what quality the deliveries should have, when the milestones are reached. The advantage of the planning system is that there are many activities that are related in a logical line, and that helps to analyze the consequences when the milestones are not reached.

All activities that will be completed are marked against each milestone in the design phase. It will then be possible to follow how far the disciplines have come. The focus is not on the activities that go through, but on those to be completed. A report that shows how each activity relates to project progress can be created. This is done on a regular (weekly) basis in the case projects.

Progress planning always starts on the date of completion and goes backwards. And so the milestones are drawn up. This methodology ensures that what shall be delivered at the completion date receive sufficient focus. PEM is not a project plan, but can reflect the plan whereas all activities in the project plan are sorted with an identifier towards milestone and quality level. The goal is to create a project plan that enables a maximum degree of harvesting of status from the BIM. Each control object is linked to the project plan through the milestones. Kvaerner has developed planning checklists that define what should be achieved by the planning system to each milestone, how many activities there are, if the activities are linked logically, if it is broken down in a way consistent with the established WBS structure etc. When the quality levels of all control objects are exported from the BIM, they can be linked to the activities in a project planning and reporting application, which in Kvaerner is Safran Project (Safran [13]). 


\section{Conclusion}

This paper has introduced control objects and how the quality levels on these are defined using different status definitions. Correct quality levels on each control object for each discipline to each milestone in the design phase can be reached by fulfilling relevant checklists. The maturity and quality of the BIM can then be visualized, through status color codes on each control object for all relevant disciplines. The control objects in the BIM can be connected to the project plan through milestones. Activities for each discipline in the project plan describe the quality on control objects that must be reached to each of the milestones in the design phase. This can be expressed through quality levels on the control objects. This makes it possible to aggregate status of activities (related to a milestone) directly from the BIM, through quality levels on relevant control objects.

The common denominator for the connection between plan and BIM are milestones. All milestones and control objects with quality levels are dated in the planning system. All activities in the project plan are linked to the milestones. The quality level on each control object (for each discipline) on a given milestone can visualize whether the project (through disciplines) is ahead of, on or behind schedule. Instead of manually reporting, progress towards milestones in the project plan can be reported directly from the BIM.

This paper has described how we can report progress towards milestones in the project plan, as defined in a project execution model (PEM), directly from a 3D design environment (BIM), based on experiences from case projects at Kvaerner. According to Mejlænder-Larsen [7] the oil and gas industry and construction industry have a high degree of similarity related to project execution, and more specifically on PEM and BIM, which makes the findings in Kvaerner relevant to adapt towards the construction industry. The focus for further research will be to discuss the findings theoretically and to develop concepts (models and frameworks) to be able to use the findings identified in this paper in the construction industry.

\section{Acknowledgements}

The author would like to thank Kvaerner for access to case projects and resources, coordinated through Tom Henningsen and Bjørn Lindal. The author would also like to thank the supervisors at Norwegian University of Science and Technology (NTNU), Professor Tore Brandstveit Haugen (Department of Architectural Design and Management) and Professor Ole Jonny Klakegg (Department of Civil and Transport Engineering), for valuable feedback. The author would like to thank supervisor Håkon Sannum at Multiconsult for expert advices.

\section{References}

[1] Kvaerner. Application Factsheets. 2012.

[2] NBIMS. National Building Information Modeling Standard. National Institute of Building Sciences, 2007. 
[3] Sacks R, Radosavljevic M, Barak R. Requirements for building information modeling based lean production management systems for construction. Automation in Construction. 2010; 19(5): pp. 641-55.

[4] Chen L, Luo H. A BIM-based construction quality management model and its applications. Automation in Construction. 2014; 46: pp. 64-73.

[5] Wang W-C, Weng S-W, Wang S-H, Chen C-Y. Integrating building information models with construction process simulations for project scheduling support. Automation in Construction. 2014; 37: pp. 68-80.

[6] Sacks R, Treckmann M, Rozenfeld O. Visualization of work flow to support lean construction. Journal of Construction Engineering and Management. 2009; 135(12): pp. 1307-1315.

[7] Mejlænder-Larsen Ø. Generalising via the Case Studies and Adapting the Oil and Gas Industry's Project Execution Concepts to the Construction Industry. Procedia Economics and Finance. 2015; 21(0): pp. 271-278.

[8] AkerSolutions. PEM NB Terms/Definitions and Abbreviations. 2009 Contract No.: PEM-NB-G-2.

[9] AVEVA. AVEVA PDMS 2015 (cited $7^{\text {th }}$ May 2015). Available from: http://www.aveva.com/en/Products_and_Services/Product_Finder/

AVEVA_PDMS.aspx

[10] AkerSolutions. PEM new-build method statement. 2011.

[11] RIBA. RIBA Plan of Work. 2013.

[12] Kvaerner. Kvaerner PEM Method Statement. 2012.

[13] Safran. Safran Project 2015 (cited 19 ${ }^{\text {th }}$ May 2015). Available from: http://www.safran.com/products/safran-project/ 